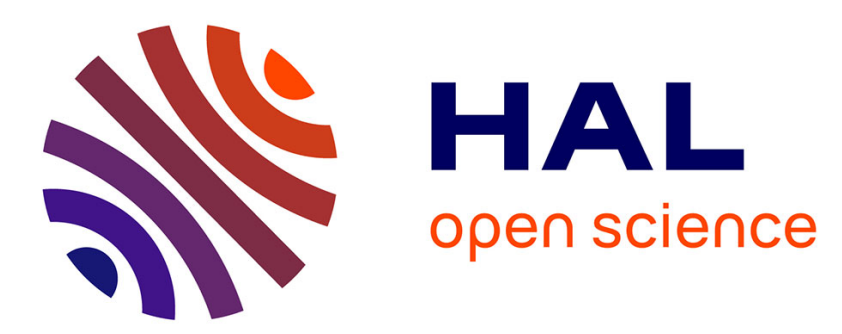

\title{
DEVS Modelling and Simulation of Human Social Interaction and Influence
}

\author{
Youssef Bouanan, Grégory Zacharewicz, Bruno Vallespir
}

\section{To cite this version:}

Youssef Bouanan, Grégory Zacharewicz, Bruno Vallespir. DEVS Modelling and Simulation of Human Social Interaction and Influence . Engineering Applications of Artificial Intelligence, 2016, 50, pp.8392. 10.1016/j.engappai.2016.01.002 . hal-01310925

\section{HAL Id: hal-01310925 \\ https://hal.science/hal-01310925}

Submitted on 3 May 2016

HAL is a multi-disciplinary open access archive for the deposit and dissemination of scientific research documents, whether they are published or not. The documents may come from teaching and research institutions in France or abroad, or from public or private research centers.
L'archive ouverte pluridisciplinaire HAL, est destinée au dépôt et à la diffusion de documents scientifiques de niveau recherche, publiés ou non, émanant des établissements d'enseignement et de recherche français ou étrangers, des laboratoires publics ou privés. 


\section{DEVS modelling and simulation of human social interaction and influence}

Article in Engineering Applications of Artificial Intelligence · April 2016

Impact Factor: 2.21 · DOI: 10.1016/j.engappai.2016.01.002

READS

44

3 authors:

Youssef Bouanan

University of Bordeaux

9 PUBLICATIONS 7 CITATIONS

SEE PROFILE

Bruno Vallespir

University of Bordeaux

149 PUBLICATIONS 852 CITATIONS

SEE PROFILE
Gregory Zacharewicz

University of Bordeaux

91 PUBLICATIONS 229 CITATIONS

SEE PROFILE 


\title{
DEVS Modelling and Simulation of Human Social Interaction and Influence
}

\author{
Youssef Bouanan, Gregory Zacharewicz, Bruno Vallespir \\ Univ. Bordeaux, IMS, UMR 5218, F-33400 Talence, France. \\ CNRS, IMS, UMR 5218, F-33400 Talence, France. \\ youssef.bouanan@ims-bordeaux.fr,gregory.zacharewicz@ims-bordeaux.fr, \\ bruno.vallespir@ims-bordeaux.fr
}

\begin{abstract}
:
The social influence is at the centre of consideration in social science. In industrial engineering, although the enterprise has reached the age of the electronic communication, the human direct communication is not sufficiently considered even if it remains critical communication vector to transmit information. The idea is to predict some human attributes behavior that will help enterprise to make efficient decision. The research in the domain gives significant results but the impact of information on individuals within a social network is, mostly, statically modelled where the dynamic aspect is not frequently tackled. The individual's reaction to a change within an organization or ecosystem (implementation of a new system, new security instructions...etc.) is not always rationale. The opinion of individuals is influenced by information gathered about the attributes of the technology from other members of their social network. In addition, the works about modelling and simulation of the population's reactions to an event do not use explicit specification languages to support their models. A behavioural specification model is one critical missing link. Adding a clear behavioural model can help for specification verification and reuse. From literature, the DEVS formalism (Discrete EVent system Specifications) appears being general enough to represent such dynamical systems (Zeigler et al., 2000). It provides operational semantics applicable to this domain. The contributions of this work are dynamic models of individuals using low-level language to simulate the propagation of information among a group of individuals and its influence on their behaviour. In more details, we define a set of models of individuals characterized by a set of state variables and the mesh between the individuals
\end{abstract}


within a social network. Then, we introduce the information diffusion based on epidemic spreading algorithms and we transpose them into the case of the message propagation in a social network. Finally, a basic scenario is used to give a beginning of validation to our models using a platform based on DEVS formalism.

Keywords: Modeling and Simulation; DEVS Formalism; Agent-Based Model; Information Impact; Diffusion Network Model.

\section{Introduction and Motivation}

In the field of social influence, a fundamental problem is to develop a model for finding an efficient way to spread information within an informal network. It seems natural that many people are often influenced by their friends' opinions and recommendations. In industrial engineering, even if the enterprise has reached the age of the electronic communication, we assume here that the human direct communication is not sufficiently considered although it remains one critical communication vector to transmit information. This study is focussing on informal social networks that remain a critical communication vector such as coffee break or others informal meetings. The idea is to evaluate the informal communication existing in the enterprise or other organisations that takes other ways than hierarchical or process orders. These commutations can influence and change the members' opinion in the field of organizational management (for instance the implementation of a new information system). Therefore, the behaviours of individuals and user groups have to be modelled and integrated into simulators to get more realistic results. The research in social science domain gives significant results but the impact of information on individuals within a social network is, mostly, statically modelled where the dynamic and sequential aspects are not frequently tackled.

Social interaction plays an important role in studying the propagation of information, innovation, ideas, and influence among its members. When an event appears - for example, the use of cell phones among students, the adoption of a new information system within the enterprise, or the rise of a political movement in an unstable society - it can either die out quickly or makes significant inroads into a population. Network diffusion process allows us to understand the dynamics and the propagation of information in social network. These 
networks can consist of individuals, group of person or organizations. The interactions could be done by physical contact, remote collaboration, any types of social meetings or some forms of verbal or written communication depending on the situations. The diffusion studies are numerous because diffusion phenomena are discussed in several disciplines: computer science (computer virus, information diffusion in a social network) (Girvan et al., 2002), biology (epidemics) (Cauchemez, 2011), physics, etc. In our case we diffuse a message enclosed in a data packet. This packet contains several pieces of information which influence the reaction of the individual and time life of the packet. The intention is to go further than most of current models which generally reduce the individuals as simple obstacles or information transmitters without enough nuances in their behaviour and the influence they can have on a message.

The choice of an appropriate modelling technique to study the behaviour of complex systems is very important (Robinson, 2004). To inform the choice of modelling technique, the relevant literature spanning the fields of Economics, Social Science, Psychology, Retail, Marketing, Operational Research, Artificial Intelligence, and Computer Science has been reviewed. Agent-based Modelling and Simulation (ABMS) play an important role in the field of computational social science. It is a method of computational representation of individual interactions from which social patterns emerge; the latter is a method that affords (dynamic) structural analysis of (socio) structural patterns. Agent-Based Modelling (ABM) shows microlevel processes that affect macro level outcomes. The macro level behaviour is not explicitly modelled; it emerges from the micro-decisions made by the individual entities (Pourdehnad et al., 2002). Today, Agent-Based Models (ABMs) are mostly implemented as object-oriented computer programs. They consist of autonomous agents that can be perceived as computer programs themselves. Agents have in principal three features: they behave and interact according to a given set of rules, possess cognitive capabilities to process information, and constitute their own environment (Ferber, 1999). In this study we use the Discrete EVent Systems specifications (DEVS) formalism to represent agents. DEVS has the interest to be timed, highly modular and hierarchical for the description of reactive systems. This low-level language permits a full control of the agents' models and it promotes modularity and reusability. 
A cellular automaton is a very simple abstraction invented in the 1940s by John von Neumann to study self-reproducing systems (Von Neumann and Burks, 1966). Since then, Cellular Automata (CA) have been widely used to reproduce and better understand the dynamics of complex phenomena found in the real world, especially when these phenomena only require very simple programming to be reproduced digitally. Despite their high ability to describe complex phenomena, however, CA still suffer from a low ability in supporting structural rules at various levels. In this article, we recall an extension of the CA that compensates this missing structure by means of another simple but equally powerful mathematical abstraction called DEVS. First, we give a comprehensive description of the resulting Cell-DEVS formalism. Then, we demonstrate the ability of this formalism to express the phenomena of social influence. In more detail, this paper will participate in the definition of a set of models that addresses the entities, the structure of a population and the social interaction. We begin by recalling the DEVS formalism and the CD++ toolkit. In addition, we provide a model of individual characterized by a set of attributes using DEVS. Finally, we present a basic scenario of message diffusion over a population to validate our models using a platform based on DEVS formalism.

\section{Background and related work}

In this section we introduce the concepts used along the article and we analyse some related works from the literature. We first introduce the DEVS formalism and its extension (focusing on Cell-DEVS). Then we describe some DEVS simulation tools and we focus on CD++, the DEVS simulation environment in which the actual implementation of our work is based on. Finally, we analyse some related work on the diffusion model.

\subsection{DEVS formalism}

The DEVS formalism for modelling and simulation (Zeigler et al., 2000) is based on discrete events modelling. It provides a framework with mathematical concepts based on the sets theory and systems theory to describe the structure and the behaviour of a system. With DEVS, there is an explicit separation between a model and its simulator. Once a model is defined, it is used to build a simulator (i.e. a device able to execute the model's instructions). DEVS proposes two kinds of models: the atomic models, which describe behaviour, and the 
coupled models which describe a structure and hierarchy. DEVS has been used as a simulation paradigm for various systems (Sarjoughian et al., 2001). In our work, we use it to model human behaviour.

The DEVS formalism has numerous advantages. We can classify them into two aspects: theoretical and applicable perspectives (Figure 1). In the theoretical perspective, the DEVS formalism allows the building of a very complex model by connecting different DEVS models, either atomic or coupled models, in a hierarchical manner (Figure 1 Left Circle). Besides, the formalism provides simple but clear semantics for the basic model behavior (Figure 1 Top Circle). It can specify a specific state at any point of time as well as connect with other models with I/O events which are caused by state transitions. In addition it permits structural coupling to assembly DEVS and DEVS extension so it encourages interoperability (Figure 1 Right Circle). It is well knows and several implementations exist (Figure 1 Down Circle).

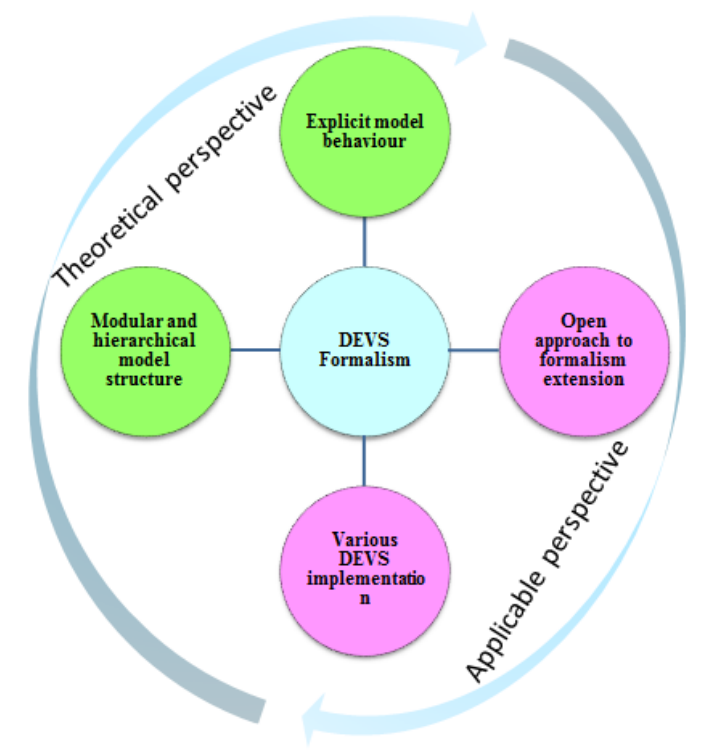

Figure 1 : Advantages of DEVS formalism from two perspectives

\subsubsection{Formal specification of an atomic DEVS model}

An atomic DEVS model is defined as:

$$
\mathrm{AM}=<\mathrm{X}, \mathrm{Y}, \mathrm{S}, \text { ta }, \delta_{\text {int }}, \delta_{\text {ext }}, \lambda>
$$


- $\mathrm{X}$ : is the set of input events;

- $\mathrm{Y}$ : is the set of output events;

- $\mathrm{S}$ : is the set of sequential states (or also called the set of partial states);

- Ta: is the time advance function which is used to determine the lifespan of a state;

- $\delta_{\text {ext }}$ is the external transition function which defines how an input event changes a state of the system;

- $\delta_{\text {int: }}$ is the internal transition function which defines how a state of the system changes internally (when the elapsed time reaches to the lifetime of the state);

- $\lambda$ : is the output function. It defines how a state of the system generates an output event (when the elapsed time reaches to the lifetime of the state).

The semantics for this definition is given as follows. At any time, a DEVS atomic model is in a state $s \in \mathrm{S}$. In the absence of external events, the model stays in this state for the duration specified by $\operatorname{ta}(\mathrm{s})$. When the elapsed time $\mathrm{e}=\mathrm{ta}(\mathrm{s})$, the state duration expires and the atomic model sends the output $\lambda(\mathrm{s})$ and performs an internal transition to a new state specified by $\delta_{\text {int }}(\mathrm{s})$. Transitions that occur due to the expiration of ta(s) are called internal transitions.

However, state transition can also happen due to the arrival of an external event which will place the model into a new state specified by $\delta_{\text {ext }}(\mathrm{s}, \mathrm{e}, \mathrm{x})$; where $\mathrm{s}$ is the current state, e is the elapsed time, and $\mathrm{x}$ is the input value. The time advance function $\operatorname{ta}(\mathrm{s})$ can take any real value from 0 to $+\infty$. A state with ta(s) value of zero is called transient state, and on the other hand, if $\operatorname{ta}(\mathrm{s})$ is equal to $+\infty$ the state is said to be passive, in which the system will remain in this state until receiving an external event.

\subsubsection{Formal specification of a coupled DEVS model}

The classic DEVS coupled model can be described as:

$$
\mathrm{N}=\langle\mathrm{X}, \mathrm{Y}, \mathrm{D},\{\mathrm{Mi}\},\{\mathrm{Ii}\},\{\mathrm{Zi}, \mathrm{j}\}, \text { select }>
$$

\subsubsection{DEVS extensions}

The DEVS formalism supports an open approach to formalism extension, allowing the researcher to explore new extended or specialized formalism (Zeigler and Vahie, 1993). 
These extensions facilitate the development of models for various applications in many different domains such as biology, engineering, and sociology. For example, Barros (1996) proposed the Dynamic Structure DEVS (DSDEVS) formalism which allows changes in model structure during execution. Chow (1996) proposed the Parallel DEVS (P-DEVS) for parallel execution benefits. Hong et al. (1997) proposed the Real Time DEVS (RT-DEVS) for executing DEVS models within a real-world environment. From a network modelling perspective, Uhrmacher (2007) proposed Multi-Level-DEVS (ml-DEVS) which supports an explicit description of macro and micro level and Wainer and Giambiasi (2002) proposed the Cell-DEVS formalism which is a combination of cellular automata and DEVS that allows the implementation of cellular models with timing delays. Among them, we will use Cell-DEVS in this study to model and simulate the dissemination and the impact of information.

Wainer and Giambiasi $(2001,2002)$ proposed the Cell-DEVS formalism to describe cell spaces as discrete-event models where each cell is represented by a DEVS basic model component to combine the advantages of CA and DEVS methodologies in a systematic way. Using a modular interface, each DEVS basic model can communicate with its neighboring cells in the cell space, as well as other models outside of the cell space. Cell-DEVS is a combination of CA and DEVS that allows the implementation of cellular models with timing delays. A Cell-DEVS model is defined as a lattice of cells holding a state variable and a computing apparatus to update the cell state. This is done using the present cell state and a set of inputs coming from cells in the neighborhood. Cell-DEVS improves the execution performance of cellular models by using a discrete-event approach. It also enhances the cell's timing definition by making it more expressive. Each cell is defined as an atomic model using timing delays. It can be later integrated to a coupled model by putting together a number of cells interconnected by a neighborhood relationship as showed in Figure 2. 


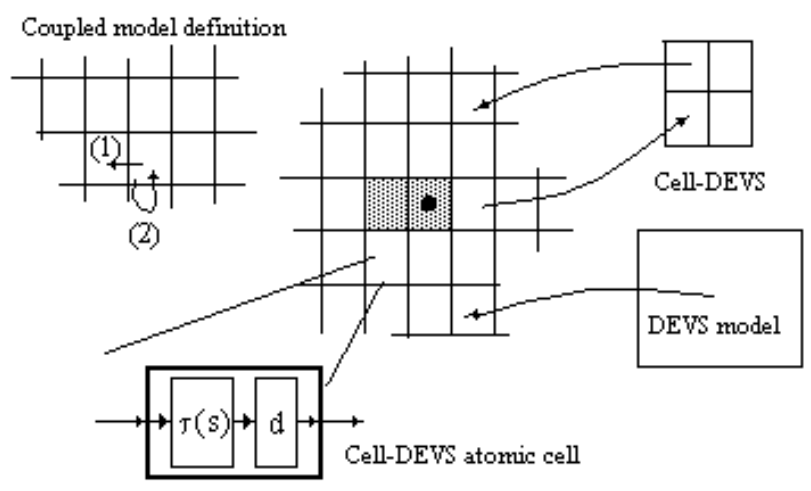

Figure 2: Informal Description of Cell-DEVS

Each cell uses $\mathrm{N}$ inputs to compute its next state. These inputs, which are received through the model's interface, activate a local computing function $(t)$. A delay $(d)$ can be associated with each cell. The state $(s)$ changes can be transmitted to other models, but only after the consumption of this delay. Two kinds of delays can be defined: transport delays model a variable commuting time (every state change is transmitted), and inertial delays, which have pre-emptive semantics (scheduled events can be discarded).

\subsubsection{DEVS tools}

The DEVS formalism presents an explicit separation between model specification and its implementation (or simulation development). In other words, implementing DEVS models is easily achievable by using an implementation framework supporting the DEVS formalism, such as CD++ (Wainer, 2002), a framework which uses C++, JDEVS (Filippi et al., 2004) and DEVSJAVA (ACIMS, 2012) which both (JDEVS and DEVSJAVA) use JAVA, PythonDEVS (Bolduc et al., 2002) which is written in Python and VLE (virtual laboratory environment) which is a multi-modelling and simulation platform developed in C++ (Quesnel et al., 2009).

Several simulators for DEVS have been implemented. They are built with various Objectoriented languages (OOL) and even for those which use the same language, the simulation algorithms are different. The proposed DEVS and Cell-DEVS models in this study will be implemented in the $\mathrm{CD}++$ simulator. $\mathrm{CD}++$ is a modelling and simulation toolkit that implements DEVS and Cell-DEVS theory (Wainer, 2002). It has been used to solve a variety of complex applications (Wainer et al., 2010; Wainer, 2006). The tool allows models to be 
defined according to the specifications introduced in the previous section. DEVS atomic models can be incorporated into a class hierarchy in $\mathrm{C}++$, while coupled models are defined using a built-in specification language. The tool also includes an interpreter for a specification language that allows describing Cell-DEVS models. The model specification includes the definition of the size and dimension of the cell space, the shape of the neighbourhood and the borders. The cell's local computing function is defined using a set of rules with the form:

\section{$\{$ POSTCONDITION $\}\{$ DELAY $\}\{$ PRECONDITION $\}$.}

These indicate that when the PRECONDITION is satisfied, the state of the cell changes to the designated POSTCONDITION, whose computed value will be transmitted to other components after consuming the DELAY.

\subsection{Network diffusion process}

Social interaction plays an important role in studying the propagation of information, innovation, ideas, and influence among its members. An event will appear - for example, the use of cell phones among students, the adoption of a new information system within the enterprise - and it can either die out quickly or make significant inroads into the population. The network diffusion processes have a long history in social sciences (Rogers, 1962). With the advent of sufficient storage and computational power, this network diffusion process became an emerging research area in computer science (Domingos, 2005). Propagation models are designed to reproduce the phenomena that can be observed in social networks with applications in viral marketing, spread of disease and diffusion of ideas and innovations. Most models proposed recently are extensions from the Independent Cascade (IC) (Goldenberg et al., 2001) and the Linear Threshold models (LT) (Granovetter, 1978). The two models characterize two different aspects of social interaction. The IC model focuses on individual (and independent) interaction and influence among friends in a social network. The IC models can also be identified with the so-called Susceptible/Infective/Recovered (SIR) model for the spread of disease in a network (Bailey et al., 1975). The LT model focuses on the threshold behaviour in influence propagation, which we can frequently relate to; when enough of our friends bought a new phone, played a new computer game, or used new online social networks, we may be converted to follow the same action. 
Modelling information flow through various social networks is an active research area, with work on diffusion of innovation and technology (Bass, 2004; Brown and Reignen, 1987; Rogers, 1995), viral marketing (Leskovec et al., 2006), the spread of computer viruses (Albert et al., 2000), and the spread of diseases (Newman, 2002; Morris, 2000). Diffusion models are used to study the adoption of products and spread of innovation influence by viewing them as a process of social interactions. The diffusion of innovation theory, introduced by Everett Rogers in 1962, defines stages of product adoption process which includes knowledge, persuasion, decision, implementation, and confirmation (Rogers, 1995). With respect to marketing, ABM has often been applied to model the diffusion of innovations. For instance, several researchers (Goldenberg et al., 2009; Rahmandad and Sterman, 2008; Stephen et al., 2010) have used an agent-based approach integrated with network science to model the role of influential in diffusion. Goldenberg et al. (2010) have used ABM to explore network effects in product adoption. Garcia (2005) provides a good review of how ABM had been used in innovation research. A restricted form of ABM, known as cellular automata (CA), has also proven useful. For instance, Garber et al. (2004) and Moldovan and Goldenberg (2003) use CA to model the diffusion of innovations. Dabbaghian et al. (2012) use CA to explore how social interactions among high school adolescents can affect their eating behavior and food choice.

\section{Materials and methods}

\subsection{Human behaviour modelling}

\subsubsection{General approach}

Human Behaviour Modelling is an important area of computational science with implications not only for social sciences, but also for economics, epidemiology and other fields. Scientific literature abounds in heterogeneous and highly specialized, theoretically founded concepts of human cognition, emotion and other behaviour aspects. There are many lines of research on such models, which span several disciplines, have different goals, and often use different terminologies and various approaches. Human behaviour modelling or Human Behaviour Representation (HBR) refer to computer-based models which imitate either the behaviour of a single person or the collective actions of a team (Pew and Mavor, 1998). Nowadays, research in human behaviour modelling is discussed in a variety of application areas. The important 
fields of study are military service research (Fei et al., 2007), robotics (Kubota and Nichida, 2006), human machine interface (HMI) (Okuda et al., 2007) and some specially oriented anthropology studies (Ding, 2009). Human behaviour models are often represented by finite state machines, rules, fuzzy rules (Dorsey and Coovert, 2003) or multi-agent based modelling (Sun, 2007).

Silverman (2001) proposes a framework for integrating Performance Moderator Functions (PMF) in behavior simulation models. His framework named PMFserv is a model of an agent's cognitive-affective state and reasoning abilities that is applied to profile the traits, cognitions, and reasoning of individual leaders, followers, and others. It is composed of various behavior moderators in different categories (physiology, stress, emotions and decision) and allows the agent to adopt different coping modes depending on its internal state.

We note that human behavior modelling in various fields is evolving and tending to be more realistic. However, these models remain specified in the shape of graphs or mathematical formulas and then directly implemented and coded using classical programming languages. The disadvantage of such an approach is that there cannot be a clear distinction between modeling and simulation. It is why the applications are specific and difficult to reuse in a different context. The DEVS Formalism allows the development of robust model representation based on the concept of atomic models and on the concept of higher-level models coupling. DEVS allows a hierarchical modelling and it is totally independent of the implementation of simulators that use such system modelling.

\subsubsection{Human behaviour modelling using DEVS}

In the agent-based model, individuals or group of individuals are represented as agents. Each agent is described by a set of attributes:

- Static attributes: gender, social status, religion, age class, ethnicity, leadership and language, etc.

- Dynamic attributes (variables): opinion, interest, un/satisfied-needs, etc.

Static attributes are intrinsic or unchanged parameters, i.e., time has no effect on them.

Dynamic attributes evolve with time or events. For example, individuals can be reached or not by the information depending on its opinion and the social network configuration. 
We use the graphical DEVS notations (Song and Kim, 1994) to describe the human behavior. Figure 1 is an example that describes the message influence on the individual behavior and potentially its dissemination using the DEVS specifications. The first phase is used to configure and initialize the agent' attributes. Then, when the agent is in the "IDLE" phase and if it receives an external event from another agent on port "In_info" (In_info? Packet), it moves to "phase_0". Here, the agent calculates the trust factor $\alpha(i, j)$ between him and the sender depending on the sender's religion, language and age class. If the trust factor is higher than a predefined threshold, the receiver moves to "phase_l" else it returns to "IDLE". Then, if the message is still strong enough, the receiver moves to "phase_2" else it returns to "IDLE". This message creates an impact on the individual, and eventually its behavior depending on the agent's opinion and the relationship between him and the sender. After a period, processing_time, the receiver transmits the message on its ego-network. After the contact between receiver and sender, the receiver's variables (opinion, interest, satisfaction) change according to the message content, the sender and cultural factors.

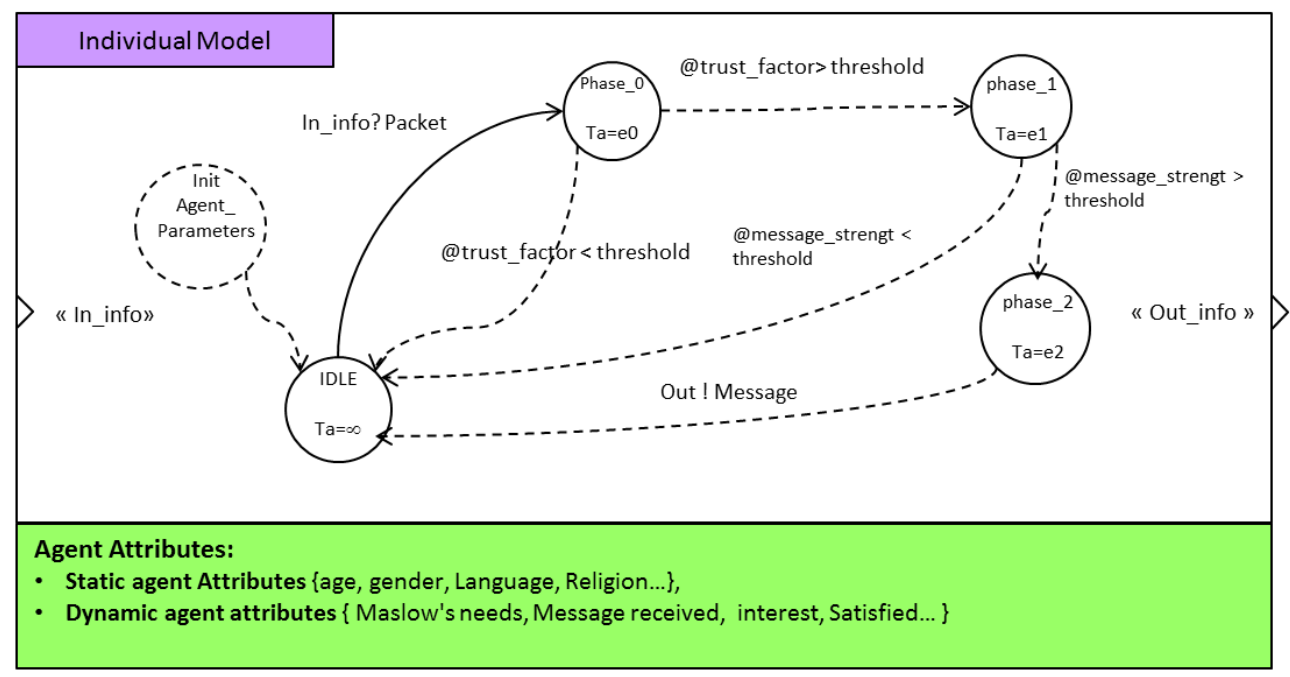

Figure 3 : Specification of individual model

\subsection{Message dissemination propagation modelling}

Propagation (dissemination or spread) models are designed to reproduce the phenomena that can be observed in social networks with applications in viral marketing, spread of disease and diffusion of ideas and innovations. 
There are clear relations between epidemic disease and information diffusion through social networks. In epidemiological modelling, the spread of diseases is often represented as the disease state of individuals, such as susceptible, infectious, and recovered, as well as possible transition and time constants for transition between states. The state and transition framework is then applied to a resolution level of the population that is appropriate for application. This may be the whole population, subpopulations, communities, or individuals. Although there are many epidemiological models available, most applications have their own specific requirements. In epidemiology, the classical disease spreading models are based upon the cycle of disease in a host: a person is first susceptible (S) to the disease. If then exposed to the disease by an infectious contact, the person becomes infected (I) (and infectious) with some probability. The disease then runs its course in that host, who is subsequently recovered (R) (or removed, depending on the virulence of the disease).

Various epidemic models have been proposed and studied over many years. Recently, researchers (Bailey, 1975 ; McCormack and Salter, 2010; Jin et al., 2013) have also applied epidemic models to the diffusion of information and influence in social networks. Both diseases and information can spread from person to person, across similar kinds of networks that connect people, and in this respect, they exhibit very similar structural mechanisms. We propose a basic analogy (Table 1) between the dissemination of information among a group of individuals and the transmission of infectious disease between the individuals themselves. In both cases, something (e.g. a message, feeling, etc.) is communicated through a given contact.

Table 1: Analytical model parameters in epidemiology versus diffusion

\begin{tabular}{|c|c|c|}
\hline $\mathbf{N}$ & Epidemiology & Information diffusion \\
\hline $\mathbf{S}(\mathbf{t})$ & $\begin{array}{c}\text { Number of individuals } \\
\text { Number of individuals }\end{array}$ \\
\hline $\mathbf{I}(\mathbf{t})$ & $\begin{array}{c}\text { Number of infectious individuals at time } \\
\mathrm{t}\end{array}$ & $\begin{array}{c}\text { Number of individuals at time } \mathrm{t} \text { that carry the } \\
\text { information }\end{array}$ \\
\hline $\mathbf{X}$ & $\begin{array}{c}\text { Contacts per unit of time and per } \\
\text { individual }\end{array}$ & Contacts per unit of time and per individual \\
\hline $\mathbf{B}$ & $\begin{array}{c}\text { Probability of transmission in a contact } \\
\text { between an infective and a susceptible }\end{array}$ & $\begin{array}{c}\text { Probability of transmission in a contact between } \\
\text { an information holder and a susceptible }\end{array}$ \\
\hline
\end{tabular}




\begin{tabular}{|c|c|c|}
\hline $\begin{array}{c}\mathbf{a}= \\
\mathbf{B x} / \mathbf{N}\end{array}$ & Infection rate & Diffusion rate \\
\hline $\begin{array}{c}\boldsymbol{\alpha}= \\
\mathbf{a} \mathbf{I}(\mathbf{t})\end{array}$ & $\begin{array}{c}\text { Force of infection: probability per unit } \\
\text { of time for a susceptible to become } \\
\text { infective }\end{array}$ & $\begin{array}{c}\text { Transmission Strength: probability per unit of } \\
\text { time for a node to receive the information }\end{array}$ \\
\hline
\end{tabular}

The diffusion model presented in this paper is motivated by concepts from existing diffusion models. The key concepts found in the SIR models used in epidemiology and the standard threshold and cascade models are reflected in the models. The SIR model divides the population into three possible categories (susceptible, infected, and removed) that reflect the status of the individuals. Susceptible are individuals who are not infected but may become infected when they get in contact with an infected individual. Infected are individuals who are carrying the disease and have the potential to spread it. Removed are individuals who have either recovered from the disease or died, and cannot spread the disease. The model assigns a disease transmission probability based on a given average rate of contact, and assumes that all individuals are equally likely to become infected. An agent is assumed to transmit messages to another agent based on the SIR model, where agents are classified into three types: A-, Band C-type. A-agent (active agent) is an agent who knows the information and is willing to make others known because the agent is itself interested by the information. B-agent (uninformed agent) has not received any message, but can be so through interaction with Aagent. C-agent (passive agent) has received a message but does not spread it. Each A-agent tries to transmit information to other uninformed agents.

Four conditions can cause the end of the simulation: (1) The individual who receives the information is a type B; (2) The strength of the message to be propagated falls below a given threshold; (3) All nodes are type C; or (4) it has been a long time (higher than a given threshold) since the scenario occurred.

\subsection{Influence and opinion change modelling}

The challenge in creating a social simulation addresses how groups or individuals influence each other, how they update their opinion and alter behaviour based on messages diffused 
from others. Many psychological results and theories address the social influence phenomenon (French, 1956; De Groot, 1974; Friedkin, 1999; Cialdini, 2001). The mechanisms of opinion change in our work are based on social influence and bounded confidence models (Hegselmann and Krause, 2002). Social influence occurs when a message emitter and a message receiver get in contact. The message emitter attempts to communicate position about an opinion to the receiver. As result of this conversation, the receiver of the message may shift her/his opinion some distance towards or away from that of the emitter's opinion. This opinion is characterized by the opinion number and the opinion confidence bounds (Figure 6). This opinion is held with a certain degree of confidence. This confidence is usually represented as either a symmetrical or asymmetrical set thresholds. This confidence impacts the ability of the receiver's opinion to change. If the emitter's opinion falls beyond the thresholds of the receiver's opinion, then the receivers will not change her/his opinion. This kind of study can be exploited to study the information diffusion in various domains such as the collaboration between several enterprises, in an organization or in the military area.

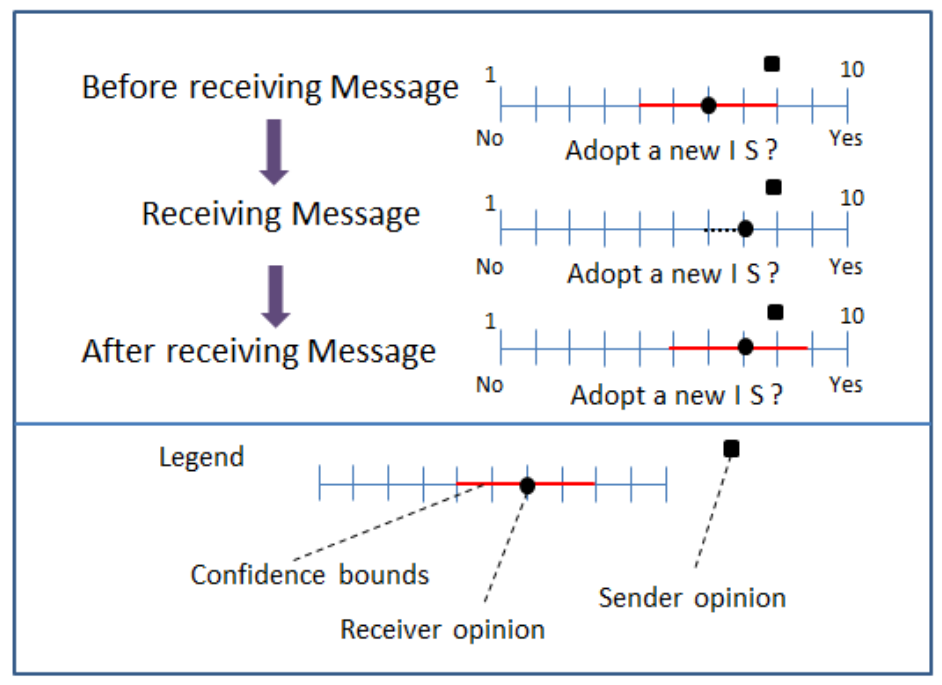

Figure 4: The mechanism of opinion change

\section{Simulation experiment}

In this section, we present a simple model of information impact (evolution of agent's opinion) using the formalism Cell-DEVS in order to validate the interest to use DEVS and Cell-DEVS in social influence modelling. These models implement limited number of data in order to facilitate its understanding in a pedagogical approach. We find encouragingly that 
such simple model with a modest amount of readily available data produces credible potential useful predictions of trends.

\subsection{Conceptual model description}

The model involves the propagation of information within a group of individuals (from infosources to info-targets) and it evaluates the affects generated by the information. Using CellDEVS, we constructed 441 agents (individuals) that represent an entire population. In CellDEVS model the population is represented by two-dimensional square grids where each cell represents an individual in a population. We assume that they are residing near the same area. Each individual, residing in $(\mathrm{i}, \mathrm{j})$ cell, is equipped with variables indicating different parameters and states of the individual; Static attributes: gender, social status, religion, age class, ethnicity, leadership, language and dynamic attributes (variables): opinion, interest, un/satisfied-needs. The state of each cell can vary depending on pre-determined rules. These rules are derived from human behaviour model described in section 2.1. Our assumption is that individuals belong to any of three states; Active (A-agent), Uninformed (B-agent) and passive (C-agent).

The protocol of opinion change is based on social influence and bounded confidence models. Social influence occurs when there is contact between an info-source (message emitter) and an info-target (message receiver). The message sender communicates a position about an opinion to the sender. As a result of this discussion, the receiver of the message may change its opinion; towards or away from that of the sender's opinion.

\subsection{Formal specification}

We now present the formal definition for the Cell-DEVS model defined in the previous paragraph. DEVS and Cell-DEVS (as CA as well) provide the advantage of a formal approach. It allows formalizing and validating the conceptual model and improves the error detection process, while reducing testing time (thus improving the quality and development costs of a simulation). DEVS permits formal specifications to be translated into executable models, facilitating the verification of the simulator and validation against as well as the controlled investigation of performance through alternative models and simulators (Shiginah, 2006). 


\subsubsection{Atomic cell-DEVS models}

The Atomic cell-DEVS specification of the agent is:

$$
\mathrm{CD}=<\mathrm{X}, \mathrm{Y}, \mathrm{I}, \mathrm{S}, \theta, \mathrm{N}, \mathrm{d}, \delta \text { int }, \delta \operatorname{ext}, \tau, \lambda, \mathrm{D}>
$$

Where

- $\mathrm{X}$ is input port to receive the package or information

- $\mathrm{Y}$ is output port to send the package or information

- $\mathrm{S}$ is the set of possible states for a given cell.

- $\mathrm{N}$ represents the von Neumann neighborhood $\{(-1,0),(0,-1),(0,1),(1,0),(0,0)$,

- $\mathrm{d}=$ transport delay, $100 \mathrm{~ms}$

- $\tau: \mathrm{N} \rightarrow \mathrm{S}$, introduced in figure 2 , is defined by following rules.

- Agent state change: After a delay, uninformed agent will become Active (from 0 to 1 ) if at least one neighbour (Von Neumann's neighbourhood) is an active agent (1).

- Opinion change: Each agent updates its opinion when it receives a message from its neighbours according to bounded Confidence (BC) mechanism.

- $O^{\prime}(x)=O(x)+\partial *(O(y)-O(x))$

Where

$O^{\prime}(x)$ Is the current opinion of the agent $(x)$ and $\partial$ is degree of influence of agent $y$.

The model we are going to exhibit portrays bounded confidence among the agents in the following sense. An agent i takes only those agents $j$ into account whose opinions differ from his own not more than a certain confidence level $\sigma(i)$ (Section 2.3).

- Message adoption: After a delay, when the agent changes its opinion it can adopt the message depending on its age class and its current opinion (opinion > 6).

- I, $\theta$, $\delta$ int, $\delta$ ext, $\lambda, D$ will be defined according to coupled Cell-DEVS definitions.

\subsubsection{Coupled cell-DEVS models}

Once the behavior of one cell is defined, they can be combined into a coupled model:

$\mathrm{M}=\langle\mathrm{I}, \mathrm{X}, \mathrm{Y}, \mathrm{Xlist}, \mathrm{Ylist}, \eta, \mathrm{N},\{\mathrm{m}, \mathrm{n}\}, \mathrm{C}, \mathrm{B}, \mathrm{Z}$, select $>$ 
Where

- $\quad$ Xlist $=\Phi$

- $\quad$ Ylist $=\Phi$

- $\eta=5$

- $\mathrm{I}=\left\langle\mathrm{P}^{\mathrm{X}}, \mathrm{P}^{\mathrm{Y}}\right\rangle$, with $\mathrm{P}^{\mathrm{X}}=\{\Phi\}, \mathrm{P}^{\mathrm{Y}}=\{\Phi\}$

- $\mathrm{N}=\{(-1,0),(1,0),(0,0),(0,-1),(0,1)\}$

- $\mathrm{m}=40 ; \mathrm{n}=40$

- $\mathrm{B}=\{\Phi\}$

- $\mathrm{C}=\{\mathrm{Cij} / \mathrm{i} \in[0,39], \mathrm{j} \in[0,39]\}$

- $\mathrm{Z}$ : defines internal and external couplings

$$
\begin{aligned}
& \mathrm{P}_{\mathrm{i}}{ }^{\mathrm{Y} 1} \rightarrow \mathrm{P}_{\mathrm{i}, \mathrm{j}+1}{ }^{\mathrm{X} 1} \\
& \mathrm{P}_{\mathrm{i} j}{ }^{\mathrm{Y} 2} \rightarrow \mathrm{P}_{\mathrm{i}-1, \mathrm{j}} \mathrm{X}^{2} \\
& \mathrm{P}_{\mathrm{ij}}{ }^{\mathrm{Y} 3} \rightarrow \mathrm{P}_{\mathrm{i}, \mathrm{j}-1} \mathrm{X} \\
& \mathrm{P}_{\mathrm{ij}}{ }^{\mathrm{Y} 4} \rightarrow \mathrm{P}_{\mathrm{i}+1, \mathrm{j}} \mathrm{X} \\
& \mathrm{P}_{\mathrm{i}} \mathrm{j}^{\mathrm{Y}} \rightarrow \mathrm{P}_{\mathrm{i}, \mathrm{j}}{ }^{\mathrm{X} 5} \\
& \mathrm{P}_{\mathrm{ij}+1} \mathrm{X} 1 \leftarrow \mathrm{P}_{\mathrm{ij}}{ }^{\mathrm{Y} 1} \\
& \mathrm{P}_{\mathrm{i}-1 \mathrm{j}}{ }^{\mathrm{X} 2} \leftarrow \mathrm{P}_{\mathrm{ij}} \mathrm{Y} \\
& \mathrm{P}_{\mathrm{ij}-1} \mathrm{X} 3 \leftarrow \mathrm{P}_{\mathrm{ij}}{ }^{\mathrm{Y}} \\
& \mathrm{P}_{\mathrm{i}+1 \mathrm{j}} \mathrm{X}^{4} \leftarrow \mathrm{P}_{\mathrm{ij}} \mathrm{Y}^{4} \\
& \mathrm{P}_{\mathrm{ij}}{ }^{\mathrm{X} 5} \leftarrow \mathrm{P}_{\mathrm{ij}}{ }^{\mathrm{Y}}
\end{aligned}
$$

- $\quad$ Select $=\{(0,-1),(0,1),(0,0),(-1,0),(1,0)\}$

The coupled model rules $\mathrm{P}$ is built as an array of atomic cells, each connected to its neighborhood. The space borders are provided with different behavior, or their cells are connected with those in the opposite border.

An agent changes its opinion (encourage or not the implementation of an information system) depending on the sender's opinion and its own current status. An agent's opinion is characterized by the opinion number, and the opinion's confidence bounds. When the agent receives an opinion, from another agent within his social network, the receiver modifies its opinion partially, in the direction of the sender's opinion. When the sender's opinion is outside the receiver's confidence bounds, the receiver ignores the sender's opinion. This model and the opinion scaling equations follow largely (Friedkin, 1999; Hegselmann and Krause, 2002) introduced in section 2.2.

We suppose that the dimension of for/against the innovation is from 0 to 10 , with 5 as indifference towards the change. Initially, the entities may be uninformed individuals or information sources with an opinion about the change affected randomly. They also have a set of thresholds around these opinions. Source nodes initiate the original message. The infosources are chosen randomly. 


\subsection{Implementation}

\subsubsection{Overview}

The CD++ environment includes facilities to build DEVS and Cell-DEVS models. Cellular models are defined using a built-in language based on the formal specifications of CellDEVS, which includes the definition of the size and dimension of the cell space, the shape of the neighbourhood, and the type of the cells that represent the borders of the bouncing area. The cell's local computing function is defined using a set of rules in the following format.

\section{POSTCONDITION DELAY \{ PRECONDITION \}}

That is, when the PRECONDITION is met, the state of the cell will change to the designated POSTCONDITION after the duration specified by DELAY. If the precondition is not met, then the next rule is evaluated until a rule is satisfied or no more rules are available. As discussed in (Wainer, 2006, 2010), this formalism allows security to be improved and development cost to be reduced thanks to the simulations.

In this section, we show the basic definitions of Cell-DEVS models by defining the behaviour of diffusion process within a group of persons. The model is specified by a $21 \times 21$ cell space using the $\mathrm{C}++$ specification language. The model defines a set of rules to control the dynamic of agent's opinion and the spread of the message, including four sets of rules for the corner and border cells each as well as a set of rules for the rest of the cell space.

\subsection{2 $\underline{\text { Settings }}$}

Our Model of the message dissemination consists of four properties:

1) We use a 2-D regular uniform lattice $(21 \times 21)$ to represent the area where innovation diffuses.

2) Each cell has a neighbourhood that includes the surrounding cells at the four possible 2D directions. Each cell in the model has a neighbourhood definition (using the relative positions to the central cell itself) that includes the central cell $(0,0)$, northwest (NW) cell $(-1,-1)$, northeast $(\mathrm{NE})$ cell $(-1,1)$, southwest $(\mathrm{SW})$ cell $(1,-1)$, and southeast $(\mathrm{SE})$ cell $(1,1)$. Each one representing an agent, which can accept 
discrete values representing various states. Each agent can be in one of two states: " 0 ", agent did not adopt the innovation, "1", agent adopted the innovation.

3) The neighbourhood is a Von Neumann neighbourhood.

4) About transition rules, the variables at each site are updated simultaneously, based on the values of the variables in their neighbors at the preceding time step, and according to the definite set of "local rules". As in this model, the agent updates its opinion when it receives a message of his neighbour. Based on the sender' opinion and its attributes (age and position in the organization) it can change its opinion converging to the sender' opinion and decide to adopt or no the innovation. In the case of this model, the irreversibility of transition is assumed, so that customers cannot "un-adopt" after adoption. Each set of rules is applied to its corresponding zone defined in the cell space.

Figure 5 represents the specification of the initialization part of this model in CD++. 


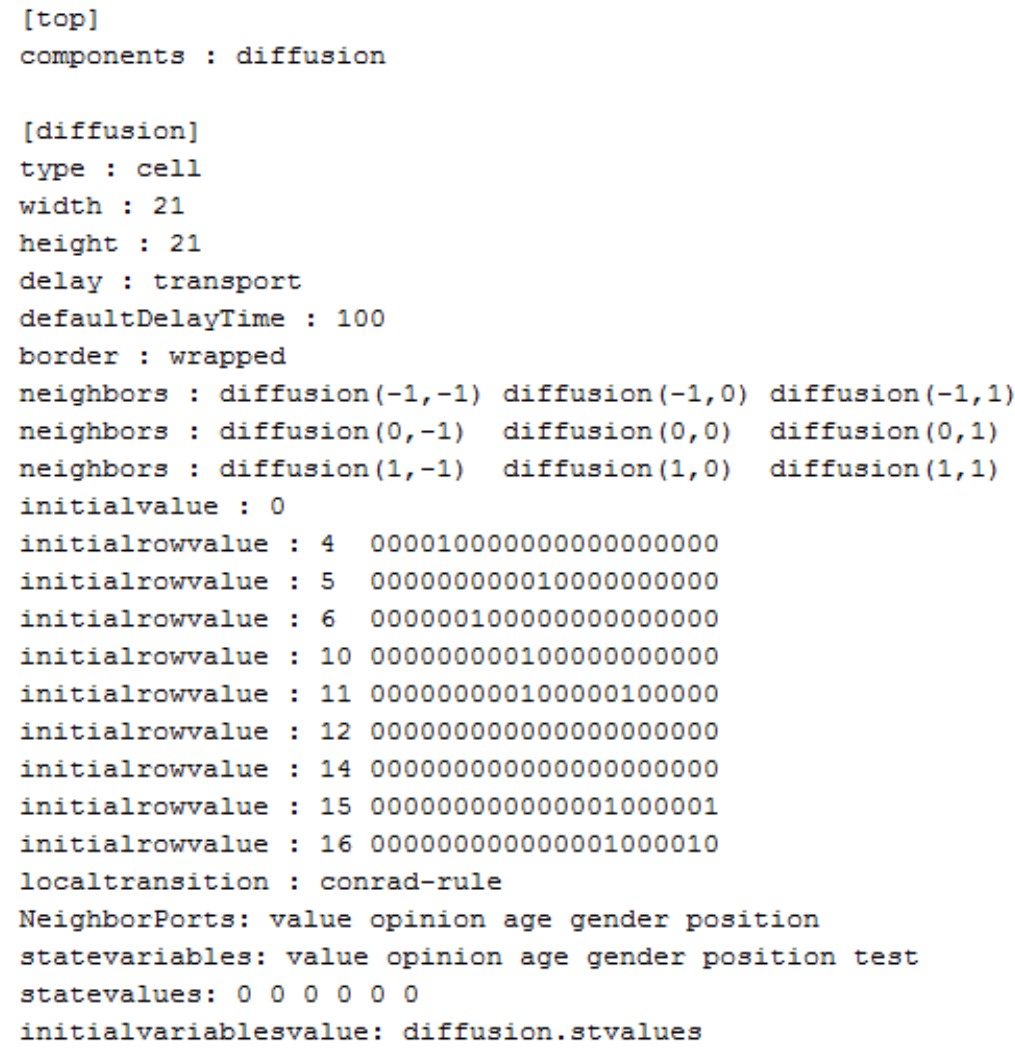

Figure 5: Diffusion model specification in $\mathrm{CD}++$

\subsection{Simulation Results}

The figure 6 shows the simulation set and results of a message propagation from info-sources to info-targets. This model represents the opinion of enterprise human resources regarding a change within the organization. Some people are pro, others are not aware about the innovation. The simulation of this model details the evolution of the individual's opinion about supporting opponents' ideas. It can be seen as a way to measure some informal information that can circulate within an enterprise. First, we generate a population of 441 individuals with some attributes; gender, position and opinion. Then, we diffuse a message from 10 sources (info-sources) represented by blue cells. In the beginning of the simulation (Figure 6A), the agents are initialized with the some degree of opinion (randomly) concerning the implementation of a new system (Red cells opinion is between 6 and 10. Orange cells represent individuals with an opinion between 3 and 6 . Yellow cell contains individual who 
are opinion between 0 and 3). When they receive the message they change their opinion based on the sender's opinion and message content. In the end (Figure 6B), we observe that the agents represented by white cells did not receive the message because they were not interest with message or because they do not share the same social networks with their neighborhood and others are changed their opinion depending on criteria presented previously.
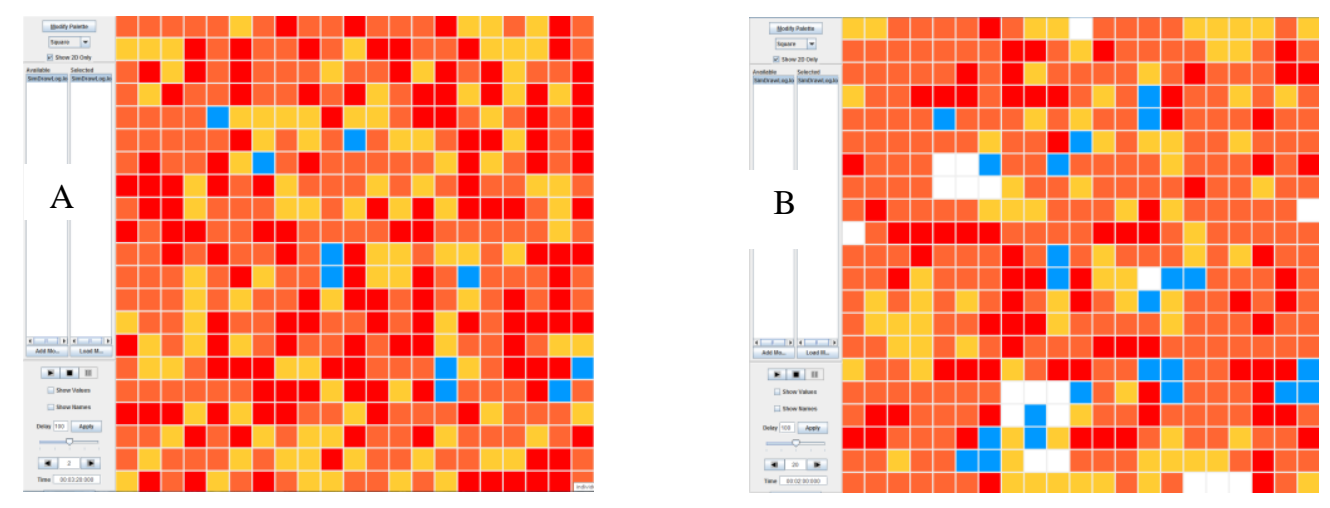

Figure 6: Simulation of human behaviour: (A) Initialization; (B) End of simulation

The individual can be opposed to the information and/or person that is transmitting the message and/or favourable to the emitter and message content. Some individuals can be reached by the information where some others not. It depends on the individuals' opinion and the configuration of the social network. This test is basic; it is based on the abstract geographical situation of individuals and it takes one dimension of a social network into account. Nevertheless, the approach already shows how hierarchical DEVS and Cell-DEVS formalism can be appropriately used to model a complex phenomenon of social influence. The results of this experiment can be exploited to reveal a communication path and to identify potential exchange of information between partners or collaborators. These informal communications can have negative or positive impacts on official orders and process actions.

These estimations done through the simulation can permit to have a more global vision on the potential impact of information in enterprise, organizations or more generally on population. This study can be used to resolve the problem of influence maximization (IM); i.e. find a set of $\beta$ initially activated nodes (info-sources) with the maximum number of activated nodes after the time step t by launching several simulations with different nodes. Then, we compare the final results to select the nodes that maximum influence or spread the information. As perspective, we are integrating progressively more variables to describe more accurately 
human behavior including social and psychological feelings and health. The inner idea is to obtain, thanks to simulation, a better understanding of the influence chain and how information can affect behavior of people and population.

At this level, our illustration model is based on fabricated data to test and simulate our design. We present a computer check of the rules and the diffusion process implemented in $\mathrm{CD}++$. Because it is based for a great part on random variable values assignment, we are aware that we must replicate the model simulation to obtain trustable tendencies. Then, the next step will be the analysis of these data to check different hypotheses.

\section{CONCLUSION AND PERSPECTIVES}

This paper introduced the formal modelling and simulation of the impact of information on individuals in a group. We have expressed how DEVS and Cell-DEVS can be appropriate and formal techniques for modelling and simulating of social influence. At the individual level, DEVS models integrate the set of attributes (e.g. gender, age and languages), variables (e.g. opinion, satisfaction degrees and interests) and behavioral functions. The individual models can transmit the message after being affected itself by the message and having potentially changed the message current strength according to several criteria. The Cell-DEVS structures use these DEVS models of human behaviour and start building on top of it connected modules to form Cell-DEVS network. In addition, the separation between the model and simulator followed by DEVS and CD++, has enabled the modeller to concentrate on building the behavioural model on one side and preparing the spreading logic on the other side using the $\mathrm{CD}++$ toolkit. The model shows a correct, even simple, human behaviour impact regarding information perception and treatment.

As a first perspective, we are integrating progressively more variables to describe more accurately human behavior including social and psychological feelings and health. The inner idea is to obtain, thanks to simulation, a better understanding of the influence chain and how information can affect behavior of people and population. This kind of simulation is promising for identifying and measuring the effects of informal information in society. Also, it opens up an interesting perspective to model human behaviour as actor of production systems. In a more general context it can serve to observe the effect of information through defined media on population. 
The main perspective remains the definition of a multi-level social network. In detail the real social network is complex, it can refer to several networks spheres from primary networks to less important networks, for e.g. to the family environment, work, , geography or any other connecting networks to transport the information from one individual to another. The final result will be the combination of several networks graphs.

One future step will consist in acquiring and using realistic data in social media for this simulation, for instance to observe the psychological effects of influence information on the population. To go further, we will also consider other experimental terrains, including domain dedicated electronic social networks.

\section{ACKNOWLEDGMENTS}

This work has been partially supported by the SICOMORES Project $\mathrm{N}^{\circ} 132936073$ funded by French DGA (Direction Générale de l'Armement). It involves the following partners: IMS university of Bordeaux, LSIS university of Marseille and MASA Group. 


\section{REFERENCES}

ACIMS, DEVSJAVA, http://www.acims.arizona.edu,2012

Albert, R., Jeong, H., \& Barabasi, A. (2000). Error and attack tolerance of complex networks. Nature, 406(6794), 378-382.

Bailey, N. T. et al. 1975. The mathematical theory of infectious diseases and its applications. Charles Griffin \& Company Ltd.

Barros FJ. (1996).The dynamic structure discrete event system specification formalism. Trans Soc Comput Simul Int; 13(1): 35-46.

Bass, F. (2004). A new product growth for model consumer durables. Management Science, 50(Supplement 12), 1825-1832.

Brown, J., \& Reignen, P. (1987). Social ties and word-of-mouth referral behaviour. Journal of Consumer Research, 14(3), 350-362.

Cauchemez, S., Bhattarai, A., Marchbanks, T. L., Fagan, R. P., Ostroff, S., Ferguson, N. M., ... \& Finelli, L. (2011). Role of social networks in shaping disease transmission during a community outbreak of 2009 H1N1 pandemic influenza. Proceedings of the National Academy of Sciences, 108(7), 2825-2830.

Chow ACH. Parallel DEVS: a parallel, hierarchical, modular modeling formalism and its distributed simulator. Trans Soc Comput Simul Int 1996; 13(2): 55-68.

Cialdini, R. B. (2001). Influence: Science and practice. Boston: Allyn \& Bacon.

Dabbaghian, V., Mago, V. K., Wu, T., Fritz, C., \& Alimadad, A. (2012). Social interactions of eating behaviour among high school students: a cellular automata approach. BMC medical research methodology, 12(1), 155.

DeGroot, M. H. (1974). Reaching a consensus. Journal of the American Statistical Association, 69(345), 118121.

Ding, W., Pei, L., Li, H., Xi, N., \& Wang, Y. (2009, March). The effects of time delay of internet on characteristics of human behaviors. In Networking, Sensing and Control, 2009. ICNSC'09. International Conference on (pp. 502-506). IEEE.

Domingos, P. (2005). Mining social networks for viral marketing. IEEE Intelligent Systems, 20(1), 80-82.

Dorsey, D. W. and Coovert, M. D. (2003) "Mathematical Modeling of Decision making: A Soft and Fuzzy Approach to Capturing Hard Decisions", Human Factors, Vol. 45, No. 1, pp. 117-135.

Faucher, C., Zacharewicz, G., Hamri, E.M., Frydman, C. (2012), "PSYOPS and CIMIC operations: from concepts to G-DEVS models", SpringSim (TMS), 42.

Fei, L., Ming, Y. and Guobing S. (2007), "Verification of Human Decision Models in Military Simulations", Proceedings of the IEEE 2007, The First Asia International Conference on Modeling \& Simulation, pp. 363 -368 .

Ferber, J. (1999). Multi-agent systems: an introduction to distributed Artificial Intelligence. London: AddisonWesley.

Filippi, J. B., \& Bisgambiglia, P. (2004). JDEVS: an implementation of a DEVS based formal framework for environmental modelling. Environmental Modelling \& Software,19(3), 261-274.

French Jr, J. R. (1956). A formal theory of social power. Psychological review, 63(3), 181.

Friedkin, N. E. (1999). Choice Shift and Group Polarization. American Sociological Review, 64(6), 856-875.

Friedkin, N. E., \& Johnsen, E. C. (1999). Social influence networks and opinion change. Advances in group processes, 16(1), 1-29.

Garber, T., Goldenberg, J., Libai, B., \& Muller, E. (2004). From density to destiny: Using spatial dimension of sales data for early prediction of new product success. Marketing Science, 23(3), 419-428.

Garcia, R. (2005). Uses of agent-based modeling in innovation/new product development research. Journal of Product Innovation Management, 22(5), 380-398.

Girvan, M., Callaway, D.S., Newman, M.E., and Strogatz, S.H. (2002). Simple model of epidemics with pathogen mutation. Physical Review E, 65(3), 031915.

Goldenberg, J., Han, S., Lehmann, D. R., \& Hong, J. W. (2009). The role of hubs in the adoption process. Journal of Marketing, 73(2), 1-13.

Goldenberg, J., Libai, B., \& Muller, E. (2010). The chilling effect of network externalities. International Journal of Research in Marketing, 27(1), 4-15.

Goldenberg, J., Libai, B., and Muller, E. (2001). Talk of the network: A complex systems look at the underlying process of word-of-mouth. Marketing letters, 12(3), 211-223.

Granovetter, M. (1978). Threshold models of collective behavior. American journal of sociology, 1420-1443. 
Hegselmann, R. \& Krause, U. (2002). Opinion Dynamics and Bounded Confidence Models, Analysis and Simulation. Journal of Artifical Societies and Social Simulation (JASSS), 5(3). http://jasss.soc.surrey.ac.uk/5/3/2.html.

Hegselmann, R., \& Krause, U. (2002). Opinion dynamics and bounded confidence models, analysis, and simulation. Journal of Artificial Societies and Social Simulation, 5(3).

Hong JS, Song HS, Kim TG, et al. (1997).A real-time discrete event system specification formalism for seamless real-time software development. Discrete Event Dyn Syst; 7: 355-375.

J.-S. Bolduc and H. Vangheluwe, A modeling and simulation package for classic hierarchical DEVS. Internal document for the Modelling, Simulation and Design Lab (MSDL), School of Computer Science, McGill University, 2002.

Jin, F., Dougherty, E., Saraf, P., Cao, Y., \& Ramakrishnan, N. (2013, August). Epidemiological modeling of news and rumors on twitter. In Proceedings of the 7th Workshop on Social Network Mining and Analysis (p. 8). ACM.

Kubota, N., and Nishida, K. (2006) "Prediction of Human Behavior Patterns based on Spiking Neurons", The 15th IEEE International Symposium on Robot and Human Interactive Communication (RO-MAN06).

Lee, C. (1964). Synthesis of a Cellular Universal Machine using the 29-state Model of von Neumann. Automata Theory Notes, Michigan: University of Michigan, 84-99.

Leskovec, J., Singh, A., \& Kleinberg, J. (2006). Patterns of Influence in a Recommendation Network. In Proceedings of the Pacific-Asia Conference on Knowledge Discovery and Data Mining (PAKDD).

Maslow, A. H. (1943). A theory of human motivation. Psychological review,50(4), 370.

McCormack, R., \& Salter, W. (2010). An application of epidemiological modeling to information Diffusion. In Advances in social computing (pp. 382-389). Springer Berlin Heidelberg.

Moldovan, S., \& Goldenberg, J. (2003). Cellular automata modeling of resistance to innovations: Effects and solutions. Technological Forecasting and Social Change, 71(5), 425-442.

Morris, S. (2000). Contagion. Review of Economic Studies, 67(1), 57-78.

Newman, M. E. (2002). Spread of epidemic disease on networks. Physical Review E, 66(1).

Norman Bailey. (1975) “The Mathematical Theory of Infectious Diseases and its Applications”. Griffin, London, 2nd edition.

Okuda, H. H., Suzuki, S., \& T Tsuchida, N. (2007, April). Modeling of human behavior. in man-machine cooperative system based on hybrid system framework. Robotics and Automation, 2007 IEEE International Conference on.

Pew, R. W. and A. S. Mavor (1998). Modeling Human and Organizational Behavior: Application to Military Simulations. National Academy Press. A. S. (Eds.). Washington DC, National Research Council.

Pourdehnad, J., Maani, K., \& Sedehi, H. (2002) "System dynamics and intelligent agent-based simulation: where is the synergy". In Proceedings of the XX International Conference of the System Dynamics society.

Quesnel, G., Duboz, R., \& Ramat, É. (2009). The Virtual Laboratory Environment-An operational framework for multi-modelling, simulation and analysis of complex dynamical systems. Simulation Modelling Practice and Theory, vol. 17(4), 641-653.

Rahmandad, H., \& Sterman, J. (2008). Heterogeneity and network structure in the dynamics of diffusion: Comparing agent-based and differential equation models. Management Science, 54(5), 998-1014.

Robinson, S. 2004. Simulation: The practice of model development and use. Chichester, UK: John Wiley \& Sons.

Rogers, E. (1962). Diffusion of innovativeness. NY: The Free Press of Glencoe.

Sarjoughian, H. S., Cellier, F. E., \& Zeigler, B. P. (Eds.). (2001). Discrete event modeling and simulation technologies: a tapestry of systems and AI-based theories and methodologies. Springer.

Seck, M., Frydman, C.S., Giambiasi, N., (2004), "Using DEVS for Modeling and Simulation of Human Behaviour", AIS 2004, pp. 692-698.

Shiginah, F. A. S. B. (2006). Multi-layer cellular DEVS formalism for faster model development and simulator efficiency (Unpublished doctoral thesis). University of Arizona, Tucson.

Silverman, BG, Might, R, Dubois, R, Shin, H, Johns, M, "Toward a Human Behavior Modeling Anthology for Developing Synthetic Agents," 10th Conf. On Computer Generated Forces and Behavioral Representation , SISO, May. 2001.

Song, H. S., \& Kim, T. G. (1994, December). The DEVS framework for discrete event systems control. In AI, Simulation, and Planning in High Autonomy Systems, 1994. Distributed Interactive Simulation Environments., Proceedings of the Fifth Annual Conference on (pp. 228-234). IEEE. 
Stephen, A. T., Dover, Y., \& Goldenberg, J. (2010). A comparison of the effects of transmitter activity and connectivity on the diffusion of information over online social networks. INSEAD Working Paper No. 2010/35/MKT. http://ssrn.com/abstract=1609611 Available at SSRN.

Sun, Z. (2007) "Multi-Agent Based Modeling: Methods and Techniques for Investigating Human Behaviors", Proceedings of IEEE International Conference on Mechatronics and Automation.

Uhrmacher, A.M., Ewald, R., John, M., Maus, C., Jeschke, M., and Biermann, S. (2007). Combining micro and macro-modeling in devs for computational biology. In Proceedings of the 39th conference on Winter simulation: 40 years! The best is yet to come, 871-880. IEEE Press.

Von Neumann, J., Burks, A. W. (1966). Theory of self-reproducing automata.IEEE Transactions on Neural Networks, 5(1), 3-14.

Wainer, G. (2006). Applying Cell-DEVS methodology for modeling the environment. Simulation: Transactions of the Society for Modeling and Simulation International, 82, 635-660.

Wainer, G. A., \& Giambiasi, N. (2002). N-dimensional Cell-DEVS models.Discrete Event Dynamic Systems, 12(2), 135-157.

Wainer, G., \& Giambiasi, N. (2001). Application of the Cell-DEVS paradigm for cell spaces modeling and simulation. Simulation, 71, 22-39.

Wainer, G., \& Giambiasi, N. (2002). N-dimensional Cell-DEVS models. Discrete Event Dynamic Systems, 12, $135-157$.

Wainer, G., (2002) "CD++: a toolkit to define discrete event models". Software, Practice and Experience. Vol.32, No.3. pp. 1261-1306. November

Wainer, G., Liu, Q., Dalle, O., \& Zeigler, B. P. (2010). Applying cellular automata and DEVS methodologies to digital games: a survey. Simulation \& Gaming, 41(6), 796-823.

Zeigler BP and Vahie S. DEVS formalism and methodology: unity of conception/diversity of application. In: Proceedings of the 1993 winter simulation conference, Los Angeles, CA, December 12-15, 1993, pp.573579.

Zeigler, B.P., Praehofer H., Kim T.G. (2000) “Theory of Modelling and Simulation”. Editions Academic Press, San Diego. 


\section{BIOGRAPHY}

Youssef BOUANAN is a $\mathrm{PhD}$ Student in Electrical and Computer Engineering with the Department of Systems and Computer Engineering at University of Bordeaux. His research domain includes; Model Transformation, Discrete Event Modelling (e.g., DEVS, Cell-DEVS and DS-DEVES) and Component-based software engineering. He received his Engineer degree in industrial computing and automatic from School of Engineering Science, Morocco and a M.Sc. in Enterprise System Engineering from University of Bordeaux, France.

Gregory ZACHAREWICZ is Associate Professor HDR at University of Bordeaux (IUT MP) with both competences in enterprise engineering and computer sciences. His research interests include Discrete Event modelling (e.g. DEVS), Distributed Simulation, HLA, and Workflow. He has been involved in FP7 European projects (e.g. MSEE and FITMAN). He is recently focused on Enterprise Modelling and Semantic Interoperability. He has published more than 60 papers in international journals and conferences.

Bruno VALLESPIR is full professor at University of Bordeaux, IMS laboratory. His research specialty is enterprise engineering (enterprise modeling, performance evaluation, interoperability). He is the former responsible of the Production systems group of IMS. He is member of several international working groups (IFIP, IFAC), he participated to 5 European projects, has directed more than $20 \mathrm{PhD}$ students and written more than 120 papers in journals and conferences. 